aretischer Eigensohaften von Banachverbanden mit Hilfe disjunZter Folgen, Arch. Math. 24 (1973), pp. 640-647.

[20] L. Tzafriri, Remarles on contractive projections in $L_{p}$ spaces, Israel J. Math. 7 (1969), pp. 9-15.

[21] M. Zippin, On bases in Banach spaces, Thesis: Hebrow University, Jerusalem (1968).

Received August 10, 1974

\section{Extension of real-valued $\alpha$-additive set functions}

by

R. B. KIRK and J. A. CRENSHAW (Carbondale, Ill.)

Abstract. The extension of real-valued $\alpha$-additive finite finitely additive regula. real-valued set functions from an algebra of sets to larger $\sigma$-algebras of sets is givenr The extensions are then used to obtain results on $\sigma\left(A^{*}, A\right)$ convergence of $\tau$-additive functionals on an algebra $A$ of real-valued functions on a set $X$.

Introduction. Let $A$ be a uniformly closed algebra of bounded realvalued functions on a set $X$ which separates the points of $X$ and contains the constants. Let $X$ be equipped with the $\tau_{A}$ topology which is the weakest topology on $X$ which makes each $f \in A$ continuous. In [4] the concept of $\alpha$-additive set functions on a paving $\mathscr{W}$ of subsets of $X$ was introduced to represent the $\alpha$-additive functionals in $A^{*}$, and it was indicated that the $\alpha$-additive set functions could be extended to $\alpha$-additive elements on a larger paving (this includes the fact that $\tau$-additive Baire measures in $O^{b}(X)$ can be extended to Borel measures on $X$ ). We shall establish this extension process which depends on which definition of outer measure is chosen. We then employ the extension to questions about weak, $\sigma\left(A^{*}, A\right)$, convergence of elements in $A^{*}$. We anticipate that working with a paving and that working with subalgebras of $C^{b}(X)$ will prove useful in probability theory, and in this direction we obtain a weakened form of Prochorovs' theorem. Also for subalgebras $A_{2} \subset A_{1}$ we give sufficient conditions for weak convergence of $\tau$-additive $\varphi$ in $A_{1}^{*}$ to be determined by the elements of $A_{2}$.

The authors wish to thank the referee for pointing out that our results in Section 1 should extend to exaustive functions with range a suitably endowed topological group. He also noted some of the rich literature on the subject such as done by Drewnowski [2], Sion [6] and Traynor [7]. The referee is of course correct and the authors intend to show this and that the weak additivity condition does yield the usual additivity condition in a different paper.

\$ 1. Extension. We refer the reader to [4] for many of the basic definitions and results; however, we shall indicate here some of the essential definitions.

A paving on $X$ is a family $\mathscr{W}$ of subsets which contains $\emptyset$, is closed under finite unions and intersections, and has $X=\bigcup \mathscr{W}$. The paving is full if $X \in \mathscr{W}$ and in this paper all pavings will be assumed to be full. 
Let $\mathscr{F}(\mathscr{W})$ be the algebra of subsets of $X$ generated by $\mathscr{W}$, then $M(\mathscr{W})$ will denote the set of all finite, finitely additive real-valued set functions on $\mathscr{F}$ which are regular in the sense that for each $F \in \mathscr{F}$ there is a $W \in \mathscr{W}$ such that $W \subset \mathbb{F}$ and $|m(G)| \leqslant \varepsilon$ whenever $G \in \mathscr{F}(\mathscr{W})$ with $G \subset F-W$.

For an infinite cardinal $a$, we say that $m \in M(\mathscr{W})$ is a-additive if $\inf \left\{|m|\left(W_{i}\right)\right\}=0$ for every collection $\left\{W_{i} \in \mathscr{W}^{n}: i \in I\right\}$ which is directed downward to $\emptyset$ with card $I \leqslant \alpha$. The set of $\alpha$-additive elements will be denoted by $M_{a}$ (or $M_{a}(\mathscr{W})$ ) and $\tau$ will denote the least cardinal such that $M_{\tau}=M_{\beta}$ when $\tau \leqslant \beta$. Finally, $m \in M(\mathscr{W})$ is $\alpha$-singular if there is a family $\left\{W_{i} \in \mathscr{W}: i \in I\right\}$ which is directed downward to $\varnothing$ with card $I \leqslant \alpha$ and such that $|m|(V)=|m|\left(\nabla \cap W_{i}\right)$ for all $V \in \mathscr{W}$ and all $i \in I$.

For the extension process to develop adequately it is essential we choose the proper definition of outer measure; we now give this and remark that if $X=[0,1]$, and if $m$ is the Lebesgue measure on the Borel sets $\mathscr{W}$, then $m \in M_{\sigma}(\mathscr{W})$, and $m^{*}$ agrees with the usual outer measure and the extension process yields the Lebesgue measure.

Definition 1.1. Let $\mathscr{W}$ be a full paving on $X$ and let $m \in M^{+}(\mathscr{W})$. For $A \subset X$,

$$
\begin{aligned}
m_{\alpha}^{*}(A)=\inf \left\{\sup _{W \in I} m\left(W^{c}\right): I \subset \mathscr{W}, I\right. \text { directed downward, } \\
\\
\left.\quad \operatorname{card}(I) \leqslant \alpha \text { and } A \subset \bigcup\left\{W^{c}: W \in I\right\}\right\} .
\end{aligned}
$$

Limma 1.2. Let $\mathscr{W}$ be a paving and let $m \in M^{+}(\mathscr{W})$. Then $m_{a}^{*}$ is an outer measure on $X$.

Proof. It is clear that $m_{\alpha}^{*}$ is monotone and non-negative. Let $A=\bigcup_{n=1}^{\infty} A_{n}$, and fix $\varepsilon>0$. For each $n \in \boldsymbol{N}$, let $I_{n} \subset \mathscr{W}$ be downward directed with $\operatorname{card}\left(I_{n}\right) \leqslant \alpha$ and such that

$$
A_{n} \subset \bigcup\left\{W^{c}: W \in I_{n}\right\} \text { and } m_{a}^{*}\left(A_{n}\right)+\varepsilon / 2^{n}>\sup \left\{m\left(W^{c}\right): W \epsilon I_{n}\right\} \text {. }
$$

Let $I$ denote the family of all finite intersections of members of $\bigcup\left\{I_{n}: n \in \boldsymbol{N}\right\}$. Then $I$ is directed downward with card $(I) \leqslant \alpha$ and $A \subset \bigcup\left\{W^{c}: W \in I\right\}$. Hence it follows that

$$
\begin{aligned}
m_{\alpha}^{*}(A) & \leqslant \sup \left\{m\left(W^{c}\right): W \in I\right\} \\
& \leqslant \sup \left\{m\left(W_{1}^{c} \cup \ldots \cup W_{m}^{c}\right): W_{i} \in \cup I_{n}, i=1, \ldots, m\right\} \\
& \leqslant \sup \left\{\sum_{i=1}^{m}\left(W_{i}^{c}\right): W_{i} \in \cup I_{n}, i=1, \ldots, m\right\} \\
& \leqslant \sum_{i=1}^{\infty} m_{a}^{*}\left(A_{i}\right)+\varepsilon .
\end{aligned}
$$

Since $\varepsilon>0$ was arbitrary, the proof is complete.
For $\alpha<\tau=\tau(\mathscr{W})$, let $\mathscr{W}_{\alpha}$ denote the family of all sets $W \subset X$ for which there is $J \subset \mathscr{W}$ with card $(J) \leqslant \alpha$ and $W=\bigcap J$. (That is, $\mathscr{W}_{\text {a }}$ is the family of subsets of $X$ obtained as intersections over subsets of $\mathscr{F}$ of cardinal at most $\alpha$.) For $\alpha=\tau, \mathscr{W}_{\alpha}$ will denote the family of all intersections over subsets of $\mathscr{W}$. It is clear that $\mathscr{W}_{\alpha}$ is a paving and that it is full if and only if $\mathscr{H}$ is full.

LemM 1.3. Let $\mathscr{W}$ be a full paving and let $m \in M^{+}$. Then $\Sigma\left(\mathscr{W}_{\alpha}\right)$ is a subalgebra of the $m^{*-C a r a t h e ́ o d o r y ~ m e a s u r a b l e ~ s e t s . ~}$

Proof. It is sufficient to show that if $W_{0} \in \mathscr{W}_{a}$, then $W_{0}$ is Carathéodory measurable. Fix $\varepsilon>0$ and $A \subset X$. We must show that $m_{\alpha}^{*}(A)$ $\Rightarrow m_{\alpha}^{*}\left(A \cap W_{0}\right)+m_{\alpha}^{*}\left(A \cap W_{0}^{c}\right)$. Let $I \subset \mathscr{W}$ be downward directed with card $(I) \leqslant \alpha$ and $\bigcap I=W_{0}$. Let $J \subset \mathscr{W}$ be a downward directed net with $\operatorname{cord}(J) \leqslant \alpha, A \subset \bigcup\left\{U^{c}: U \epsilon J\right\}$ and $m_{a}^{*}(A)+\varepsilon>\sup m\left(U^{c}\right)$. Since $m$ is regular, for each $W_{\epsilon} I$ there is $Z_{W} \in \mathscr{W}$ with $Z_{W} \subset W^{c}$ and $m\left(W^{c}\right)<m\left(Z_{W}\right)+$ $+\varepsilon$. Now fix $W_{x} \in I$. Since the family $\left\{U^{c}: U \in J\right\}$ is upward directed,

$$
\sup _{U \in J}\left[m\left(U^{c} \cap W_{1}\right)+m\left(U^{c} \cap W_{1}^{c}\right)\right]=\sup _{U \in J} m\left(U^{c} \cap W_{1}\right)+\sup _{U \in \mathcal{J}} m\left(U^{c} \cap W_{1}^{c}\right) .
$$

Furthermore,

$$
0 \leqslant m\left(\left(U^{c} \cap Z_{W_{1}}^{c}\right)-\left(U^{c} \cap W_{1}\right)\right)=m\left(W_{1}^{c} \cap Z_{W_{1}}^{c} \cap U^{c}\right) \leqslant m\left(W_{1}^{c}-Z_{W_{1}}\right) \leqslant \varepsilon .
$$

Hence it follows that,

$$
\begin{aligned}
m_{a}^{*}(A)+\varepsilon & \geqslant \sup _{U \epsilon J} m\left(U^{c}\right) \geqslant \sup _{U \epsilon J} m\left(U^{c} \cap W_{1}\right)+\sup _{U \epsilon J} m\left(U^{c} \cap W_{1}^{c}\right) \\
& \geqslant \sup _{U \epsilon J} m\left(U^{c} \cap Z_{W_{1}}^{c}\right)-\varepsilon+\sup m\left(U^{c} \cap W_{1}^{c}\right) \\
& \geqslant m_{a}^{*}\left(A \cap Z_{W_{1}}^{c}\right)-\varepsilon+\sup _{U \epsilon J} m\left(U^{c} \cap W_{1}^{c}\right) \\
& \geqslant m_{a}^{*}\left(A \cap W_{0}\right)-\varepsilon+\sup _{U \epsilon J} m\left(U^{c} \cap W_{1}^{c}\right) .
\end{aligned}
$$

Since $W_{1} \in I$ was arbitrary, we thus have that for all $W \in I$,

$$
m_{\alpha}^{*}(A)+2 \varepsilon \geqslant m_{a}^{*}\left(A \cap J_{0}\right)+\sup _{U \epsilon J} m\left(U^{c} \cap W^{c}\right) .
$$

Let $K=\{U \cup W: U \in J, W \in I\}$. Then $K$ is downward directed, card $(K) \leqslant \alpha$ and $A \cap W_{0}^{c} \subset \bigcup\left\{V^{c}: V \in K\right\}$. Hence it follows from (1) that,

$$
m_{\alpha}^{*}(A)+2 \varepsilon \geqslant m_{\alpha}^{*}\left(A \cap W_{0}\right)+\sup _{\substack{U \in J \\ W \in I}} m\left(U^{c} \cap W^{c}\right) \geqslant m_{\alpha}^{*}\left(A \cap W_{0}\right)+m_{\alpha}^{*}\left(A \cap W_{0}^{c}\right) .
$$

The proof is complete.

Lenna 1.t. Let $\mathscr{W}$ be a fill paving and let $m \in M_{\alpha}^{+}$. Then for each $F \in \mathscr{F}(\mathscr{H}), \quad m\left(F^{*}\right)=m_{\alpha}^{*}\left(F^{\prime}\right)$. Furthermore, the restriction of $m_{\alpha}^{*}$ to $\mathscr{F}\left(\mathscr{W}_{\alpha}\right)$ belongs to $M_{a}^{+}\left(\mathscr{W}_{a}\right)$.

Proof. For $m \in M_{a}^{+}(\mathscr{W})$, it is clear from the definition that $m\left(W^{\circ}\right)$ $=m_{\alpha}^{*}\left(W^{c}\right)$ for all $W \epsilon \mathscr{W}$. Since $m_{\alpha}^{*}$ is finitely additive on $\mathscr{F}(\mathscr{W})$ by Lemma 
2.7, $m(W)=m_{\alpha}^{*}(W)$ for all $W \epsilon \mathscr{W}$. That $m\left(F^{\prime}\right)=m_{a}^{*}(F)$ for all $F \in \mathscr{F}(\mathscr{W})$ follows from Proposition [3, 1.2]

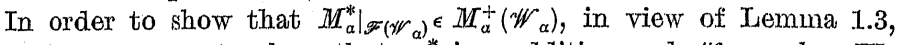
it is only necessary to show that $m_{\alpha}^{*}$ is $\alpha$-additive and $\mathscr{F}_{\alpha}$-regular. We will begin with $\alpha$-additivity which will be verified in two steps.

(1) Let $I \subset \mathscr{W}$ be downward directed with $\operatorname{card}(I) \leqslant \alpha$ and $W_{0}=\cap I$. Then

$$
m_{a}^{*}\left(W_{0}\right)=\inf _{W \in I} m(W)
$$

By definition, $m_{a}^{*}\left(W^{c}\right) \leqslant \sup _{W \in I} m\left(W^{c}\right)$. On the other hand, $W_{0} \subset W$ implies that $m_{a}^{*}\left(W_{a}^{c}\right) \geqslant m_{\alpha}^{*}\left(W^{c}\right)=m\left(W^{c}\right)$. Thus, $m_{a}^{*}\left(W_{0}^{c}\right)=\sup _{W W^{\prime} I} m\left(W^{c}\right)$, and (1) follows.

(2) If $I \subset \mathscr{W}_{a}$ is directed downward with $W_{0}=\bigcap I$, then

$$
m_{\alpha}^{*}\left(W_{0}\right)=\inf _{W \in I} m_{\alpha}^{*}(W)
$$

First note that $W_{0} \in \mathscr{W}_{a}$ since $\alpha \cdot \alpha=\alpha$. For each $W_{\epsilon} I$, let $I_{W} \subset \mathscr{W}$ be downward directed with card $\left(I_{W}\right) \leqslant \alpha$ and $W=\bigcap I_{F}$. Let $J$ denote the family of all finite intersections of the elements of the set $\bigcup\left\{I_{W}\right.$ : $W \in I\}$. Then $J \subset \mathscr{W}$ is directed downward with card $(J) \leqslant \alpha$ and $\cap J=W_{0}$. Hence, by (1), $m_{a}^{*}\left(W_{0}\right)=$ inf $m(U)$. Furthermore, if $\varepsilon>0$ is given, there is $U_{1} \epsilon J$ with $\inf m(U)+\varepsilon>m\left(U_{1}\right)$. Since $I$ is directed downward, there is $W_{1} \in I$ with $\stackrel{U_{\epsilon J}}{W_{1}} \subset U_{1}$. Hence it follows that,

$$
m_{\alpha}^{*}\left(W_{0}\right)=\inf _{U \in J} m(U)>m\left(U_{1}\right)-\varepsilon \geqslant m_{\alpha}^{*}\left(W_{1}\right)-\varepsilon \geqslant \inf _{W \in I} m_{\alpha}^{*}(W)-\varepsilon .
$$

Since $\varepsilon>0$ was arbitrary, the proof of (2) is complete.

We will now verify that $m_{a}^{*}$ is $\mathscr{W}_{\alpha}$-regular on $\mathscr{F}\left(\mathscr{W}_{a}\right)$. The proof will require three steps.

(3) Let $W_{0} \in \mathscr{W}_{\alpha}$. Then

$$
m_{\alpha}^{*}\left(W_{0}^{c}\right)=\sup \left\{m_{a}^{*}(W): W \in \mathscr{W}_{a} \text { and } W \subset W_{0}^{c}\right\} .
$$

Let $I \subset \mathscr{W}$ be directed downward with $\operatorname{card}(I) \leqslant \alpha$ and $W_{0}=\bigcap I$. Fix $\varepsilon>0$. Since $m_{\alpha}^{*}\left(W_{0}\right)=\inf _{U \in I} m(U)$ by $(1)$, there is $U_{0} \in I$ with $m_{\alpha}^{*}\left(U_{0}\right)$ $=m\left(J_{0}\right)<m_{a}^{*}\left(W_{0}\right)+\varepsilon$. This is equivalent to

$$
m_{a}^{*}\left(W_{0}^{c}\right)<m_{a}^{*}\left(U_{0}^{c}\right)+\varepsilon=m\left(U_{0}^{c}\right)+\varepsilon .
$$

Since $m$ is $\mathscr{W}$-regular, there is a $W \epsilon \mathscr{W}$ with $W \subset W_{0}^{c}$ and $m\left(U_{0}^{c}\right)<m(W)+\varepsilon$. Then $W \in \mathscr{W}_{a}, W \subset W_{0}^{c}$ and $m_{a}^{*}\left(W_{0}^{c}\right)<m\left(U_{0}^{c}\right)+\varepsilon<m(W)+2 \varepsilon$. Since $\varepsilon>0$ was arbitrary, the proof of $(3)$ is complete.
(4) Let $W_{0}, W_{1} \in \mathscr{W}_{a}$. Then

$m_{\alpha}^{*}\left(W_{1}-W_{0}\right)=\sup \left\{m_{\alpha}^{*}(W): W \in \mathscr{W}_{\alpha}\right.$ and $\left.W \subset W_{1}-W_{0}\right\}$.

Fix $\varepsilon>0$. By (3) there is a $W_{2} \in \mathscr{W}_{a}$ with $W_{2} \subset W_{0}^{c}$ and $m_{a}^{*}\left(W_{0}^{c}\right)$ $<m_{\alpha}^{*}\left(W_{2}\right)+\varepsilon$. Let $W=W_{1} \cap W_{2}$. Then $W \in \mathscr{W}_{\alpha}$ and $W \subset W_{1}-W_{0}$. Since $\left(W_{1}-W_{0}\right)-W \subset W_{0}^{c}-W_{2}$, it follows that $0 \leqslant m_{\alpha}^{*}\left(W_{1}-W_{0}\right)-m_{\alpha}^{*}(W)$ $\leqslant m_{\alpha}^{*}\left(W_{0}^{c}-W_{2}\right) \leqslant \varepsilon$. Since $\varepsilon>0$ was arbitrary, (4) follows.

(5) $m_{a}^{*}$ is $\mathscr{W}_{a}$-regular on $\mathscr{F}\left(\mathscr{W}_{a}\right)$.

This is immediate from (4) and Proposition [3, 1.2]. The proof of Lemma 1.4 is now complete.

LEMMA 1.5. Let $\mathscr{W}$ be a full paving and let $m \mathrm{e}\left(M_{a}(\mathscr{W})^{\perp}\right)^{+}$. Then $m_{a}^{*}=0$.

Proof. By $[4 ; 4.4] M_{a}(\mathscr{W})^{\perp}$ is a band so there is an increasing net $\left(m_{i}\right)$ of $\alpha$-singular elements of $M(\mathscr{W})$ with $0 \leqslant m_{i} \uparrow m$. (Since $\left(m_{i}\right)$ is increasing, it is easy to verify that $m_{i}\left(F^{\prime}\right) \uparrow m\left(F^{\prime}\right)$ for all $F^{\prime} \in \mathscr{F}(\mathscr{W})$.) Fix $\varepsilon>0$ and take $i_{0}$ with $m(X)<m_{i_{0}}(X)+\varepsilon$. Let $I \subset \mathscr{W}$ be an $a$-system with $m_{i_{0}}(X)=m_{i_{0}}(W)$ for all $W e I$. Since $0 \leqslant m-m_{i_{0}}$, it follows that, ror each $F \in \mathscr{F}(\mathscr{W}), \quad 0 \leqslant m(F)-m_{i_{0}}\left(F^{\prime}\right)=\left(m-m_{i_{0}}\right)\left(F^{\prime}\right) \leqslant\left(m-m_{i_{0}}\right)(X)<\varepsilon$. Hence

$$
0 \leqslant m_{a}^{*}(X) \leqslant \sup _{W \cdot I} m\left(W^{c}\right) \leqslant \varepsilon+\sup _{W \in I} m_{i_{0}}\left(W^{c}\right)=\varepsilon .
$$

Since $s>0$ was arbitrary, $m_{\alpha}^{*}(X)=0$, and so $m_{a}^{*}=0$.

Proposition 1.6. Let $\mathscr{W}$ be a full paving, and let $m \in M^{+}(\mathscr{W})$. Assume that $m=m_{1}+m_{2}$ where $m_{1} \in M_{a}^{+}(\mathscr{W})$ and $m_{2} \epsilon\left(M_{a}(\mathscr{W})^{\perp}\right)^{+}$. Then $m_{a}^{*}$ restricted to $\mathscr{F}\left(\mathscr{W}_{a}\right)$ belongs to $M_{a}^{+}\left(\mathscr{W}_{a}\right)$ and $m_{a}^{*}=\left(m_{1}\right)_{a}^{*}$. Hence, in particular. $m_{1}=m_{\alpha}^{*}$ on $\mathscr{F}(\mathscr{W})$.

Proof. It is easily shown that $m_{a}^{*}=\left(m_{1}\right)_{a}^{*}+\left(m_{2}\right)_{a}^{*}$. The resultris now immediate from Lemmas 1.4 and $\mathbf{1 . 5}$.

Proposition 1.7. Let $\mathscr{W}$ be a full paving, and let $m \in M_{a}^{+}(\mathscr{W})$. Then there is a unique element $\mu \in M_{a}^{+}\left(\mathscr{W}_{a}\right)$ whose restriction to $\mathscr{F}(\mathscr{W})$ is $m$. In fact, if $\lambda \in M^{+}\left(\mathscr{W}_{a}\right)$ is any element whose restriction to $\mathscr{F}(\mathscr{W})$ is $m$, then $\mu=\lambda$.

Proof. Let $\mu$ denote the restriction of $m_{a}^{*}$ to $\mathscr{F}\left(\mathscr{W}_{a}\right)$. By Proposition 1.6, $\mu \epsilon M_{a}^{+}\left(\mathscr{W}_{\alpha}\right)$ and $\mu=m$ on $\mathscr{F}(\mathscr{W})$. Now let $\lambda_{\epsilon} M^{+}\left(\mathscr{W}_{a}\right)$ and assume that $\lambda=m$ on $\mathscr{F}(\mathscr{W})$. Fix $W \in \mathscr{W}_{a}$. Let $I \subset \mathscr{W}$ be downward directed with card $(I) \leqslant \alpha$, and $W=\bigcap I$. Then $0 \leqslant \lambda(W) \leqslant \inf _{V \in I} \lambda(U)$ $=\inf _{U \in I} \lambda(U)=\mu(W)$. Since ${ }_{4} \epsilon_{4} \mathscr{W}_{a}$ was arbitrary, the $\mathscr{W}_{\alpha}$-regularity of $\lambda$ and $\mu$ guarantee that $0 \leqslant \lambda \leqslant \mu$. Since $M_{a}\left(\mathscr{W}_{\alpha}\right)$ is an ideal, it follows that $\lambda \in M_{a}^{+}\left(\mathscr{W}_{\alpha}\right)$. Hence $\lambda(W)=\inf _{U \in I} \lambda(U)=\inf _{U \in I} \mu(U)=\mu(W)$. Thus $\lambda(W)$ $=\mu(W)$ for all $W \in \mathscr{W}_{a}$. The $\mathscr{W}_{a}$-regularity of $\lambda$ and $\mu$ now imply that $\lambda=\mu$. 
Define a map $T_{\alpha}$ from $M_{a}(\mathscr{W})$ into $M_{a}\left(\mathscr{W}_{\alpha}\right)$ as follows. (We continue to assume that $\mathscr{W}$ is a full paving.) For $m \in M_{\alpha}^{+}(\mathscr{W})$, let $T_{a}(m)$ denote the restriction of $m_{\alpha}^{*}$ to $\mathscr{F}\left(\mathscr{W}_{\alpha}\right)$. Then $T_{\alpha}(m) \in M_{\alpha}^{++}\left(\mathscr{W}_{\alpha}\right)$ by Proposition 1.7. For arbitrary $m \in M_{\alpha}(\mathscr{W})$, define $T_{\alpha}(m)=T_{a}\left(m^{+}\right)-T_{\alpha}\left(m^{-}\right)$.

THEOREM 1.8. Let $\mathscr{W}$ be a full paving. The map $T_{a}$ is a Banach lattice isomorphism of $M_{\alpha}(\mathscr{W})$ onto a band in $M_{a}\left(\mathscr{W}_{\alpha}\right)$. Ifurthermore, for $\mu \in T_{a}\left[M_{a}\left(\mathscr{V}_{a}\right)\right], T_{a}^{-1}(\mu)$ is the restriction of $\mu$ to $\mathscr{F}(\mathscr{W})$.

Pro.of. It is easy to verify that $T_{\alpha}$ is linear on $M_{\alpha}^{+}(\mathscr{W})$. From this: it is immediate that $T_{\alpha}$ is a positive linear transformation on $M_{\alpha}(\mathscr{W})$. If $T_{a}(M)=0$, then $T_{\alpha}\left(m^{+}\right)=T_{\alpha}\left(m^{-}\right)$so that $\mathrm{m}^{+}=m^{-}$by Proposition 1.7. Hence if $T_{a}(m)=0$, then it follows that $m=0$. Finally, note that if $0 \leqslant T_{a}(m)$, then $0 \leqslant m$ since $m$ is the restriction of $T_{a}^{\prime}(m)$ to $\mathscr{F}(\mathscr{W})$.

In order to verify that $T_{\alpha}$ is lattice preserving, it is sufficient to show that $\left|T_{a}(m)\right|=T_{a}(|m|)$ for all $m \in M_{a}(\mathscr{W})$. Since $T_{a}$ is a positive transformation, $\left|T_{a}(m)\right| \leqslant T_{a}(|m|)$. Let $m^{\prime}$ denote the restriction of $\left|T_{a}(m)\right|$ to $\mathscr{F}(\mathscr{W})$. Then $0 \leqslant m^{\prime} \leqslant|m|$. It is immediate from this that $m^{\prime} \in M(\mathscr{W})$. Since $M_{\alpha}(\mathscr{W})$ is an ideal, it now follows that $m^{\prime} \in M_{\alpha}(\mathscr{W})$. By Proposition 1.7, $T_{\alpha}\left(m^{\prime}\right)=\left|T_{\alpha}(m)\right|$. Thus $m \leqslant m^{\prime}$ and $-m \leqslant m^{\prime}$ so that $\left|T_{\alpha}(m)\right|$ $=T_{a} m^{\prime} \geqslant T_{\alpha}|m|$. Hence $\left|T_{a}(m)\right|=T_{\alpha}(|m|)$. Moreover, $\left\|T_{a}(m)\right\|=\left|T_{\alpha}(m)\right|(X)$ $=T_{a}(|m|)(X)=|m|(X)=\|m\|$ so that $T_{a}$ is norm-preserving.

We will now show that the image of $M_{\alpha}(\mathscr{W})$ under $T_{\alpha}$ is an ideal in $M_{a}\left(\mathscr{W}_{a}\right)$ : Since $T_{\alpha}$ is lattice preserving, $T_{a}\left[M_{a}(\mathscr{W})\right]$ is a Riesz subspace of $M_{a}\left(\mathscr{W}_{a}\right)$. Now let $\lambda \in M_{a}\left(\mathscr{W}_{a}\right)$ satisfy $0 \leqslant \lambda \leqslant T_{a}(m)$ for some $m \in M_{a}(\mathscr{W})$. Let $m^{\prime}$ be the restriction of $\lambda$ to $\mathscr{F}(\mathscr{W})$. Then $0 \leqslant m^{\prime} \leqslant m$ so that $m^{\prime} \in M_{a}(\mathscr{W})$ since $M_{a}(\mathscr{H})$ is an ideal. But then $T_{\alpha}\left(m^{\prime}\right)=\lambda$ by Proposition 1.7. Hence $\lambda \in T_{\alpha}\left[M_{a}(\mathscr{H})\right]$.

Finally, in order to demonstrate that $T_{a}\left[M_{a}(\mathscr{W})\right]$ is a band, $\operatorname{let}\left(T_{a}\left(m_{i}\right)\right)$ be an upward directed net in $\left(T_{a}\left[M_{a}(\mathscr{W})\right]\right)^{--}$with $T_{a}\left(m_{i}\right) \uparrow \mu \epsilon M_{\alpha}\left(\mathscr{W}_{a}\right)$. Let $m$ denote the restriction of $\mu$ to $\mathscr{F}(\mathscr{W})$. Since $m_{i}(X)=T_{\alpha}\left(m_{i}\right)(X) \uparrow$ $\uparrow \mu(X)=m(X)$, if $\varepsilon>0$ is fixed, there is an $i_{0}$ with $m(X)<m_{i_{0}}(X)+\varepsilon$. Thus $m(F) \leqslant m_{i_{0}}\left(F^{\prime}\right)+\varepsilon$ for all $F \in \mathscr{W}$. It now follows immediately that $m$ is $\mathscr{W}$-regular so that $m \in M(\mathscr{W})$. Since $m_{i}\left(I^{\prime}\right) \uparrow m\left(W^{\prime}\right)$ for all $F^{\prime} \in \mathscr{F}(\mathscr{W})$ and since $\left(m_{i}\right)$ is directed upward, $m_{i} \uparrow m \in M(\mathscr{W})$. Since $M_{a}(\mathscr{W})$ is a band, $m \in M_{a}(\mathscr{W})$. Finally, by Proposition 1..7, $T_{a}(m)=\mu$. The proof is complete. shows.

The map $T_{\alpha}$ is not onto $M_{\alpha}\left(\mathscr{W}_{\alpha}\right)$ in general as the following example

ExanIPLE. Let $X=[0,1]$. Define $W_{0}=[0,1)$ and $W_{n}=[1-1 / n, 1]$ for $n \in \boldsymbol{N}$. Let $\mathscr{W}$ be the smallest paring on $X$ containing $\left\{W_{i}: i=0,1, \ldots\right\}$. (Thus $W=\left\{X, W_{0}, \emptyset\right\} \cup\left\{W_{n}: n \in \boldsymbol{N}\right\} \cup\left\{W_{0} \cap W_{n}: n \in \boldsymbol{N}\right\}$. ) Note that $\mathscr{W}$ is a full paving.

For $F \in \mathscr{F}\left(\mathscr{W}_{\sigma}\right) \quad\left(\sigma=\mathscr{N}_{0}\right)$, define $\lambda(F)=1$ if $1 \in F^{\prime}$ and $\lambda(\boldsymbol{F})=0$ if $1 \notin F$. Then $\lambda \in M_{\sigma}\left(\mathscr{W}_{\sigma}\right)$ as is easily seen. If $m$ denotes the restriction of $\lambda$ to $\mathscr{F}(\mathscr{W})$, then $m \notin M(\mathscr{W})$ since $m$ is not $\mathscr{W}$-regular. (Indeed, $m\left(W_{0}^{c}\right)=1$, but $\sup \left\{m(W): W \in \mathscr{W}\right.$ and $\left.W \subset W_{0}^{c}\right\}=0$.)

The following gives a simple condition on $\mathscr{W}$ which guarantees that $T_{a}$ is onto $M_{a}\left(\mathscr{W}_{a}\right)$. (Note that the family $\mathscr{Z}$ of all zero sets on a topological space satisfies the condition, and this accounts for the fact that every Baire measure on a topological space is $\mathscr{Z}$-regular.)

THEorem 1.9. Let $\mathscr{W}$ be a full paving. Assume that if $W_{0} \in \mathscr{W}$, then there is a sequence $\left(W_{n}\right)$ in $\mathscr{W}$ with $W_{0}^{c}=\bigcup_{n=1}^{\infty} W_{n}$. Then for every infinite cardinal $\dot{\alpha}, T_{\alpha}$ maps $M_{a}(\mathscr{W})$ onto $M_{a}\left(\mathscr{W}_{\alpha}\right)$.

Proof. Let $\mu \in M_{a}^{+}\left(\mathscr{W}_{\alpha}\right)$ and let $m$ denote the restriction of $\mu$ to $\mathscr{F}(\mathscr{W})$. Then $m$ is a non-negative, finite, finitely-additive function on $\mathscr{F}(\mathscr{W})$. All that need be verified is that $m$ is $\mathscr{W}$-regular. Hence let $W_{0} \subset \mathscr{W}$ and choose an increasing sequence $\left(W_{n}\right)$ in $\mathscr{W}$ with $W_{0}^{c}=\bigcup\left\{W_{n}: n \in \boldsymbol{N}\right\}$. Since $\mu$ is $a$-additive, $m\left(W_{0}^{c}\right)=\lim m\left(W_{n}\right) \leqslant \sup \{m(W): W \in \mathscr{W}$ and $\left.W \subset W_{0}^{c}\right\}$. By Proposition $[4 ; 1.4(3)]$, it follows that $m$ is $\mathscr{W}$-regular.

§ 2. Applications of the extension. In this section, we wish to apply the extension theorems to obtain certain results on weak convergence in $A^{*}$ where $A$ is a uniformly closed algebra of bounded real-valued functions on $X$ which separates the points of $X$ and contains the constants. We shall denote the paving of zero sets of $A$ by $\mathscr{Z}(A)$. If $\mathscr{W}$ is a full paving on $X$, then a standard representation of $A^{*}$ is an isometric isomorphism $I$ of $A^{*}$ onto $M(\mathscr{W})$ such that $I_{\varphi}(W)=\inf \left\{\varphi(f): f \in A, \chi_{W} \leqslant f\right\}$ for all $W \in \mathscr{W}$.

LEMMa 2.1. Let $A_{1}, A_{2}$ be algebras on $X$ with $A_{2} \subset A_{1}$ and let $\tau_{A_{1}}=\tau_{A_{2}}$. Let $\mathscr{W}_{1}, \mathscr{W}_{2}$ be two full pavings of closed sets in $X$ which are bases for the $\tau_{A_{i}}$ closed sets. Let $\varphi \epsilon\left(A_{1}^{*}\right)_{\tau}^{+}$. If $M\left(\mathscr{W}_{i}\right)$ represents $A_{i}^{*}, i=1,2$, and $m \in M_{\tau}^{+}\left(\mathscr{W}_{1}\right)$ represents $\varphi$ while $\mu \in M_{\tau}^{+}\left(\mathscr{W}_{2}\right)$ represents $\varphi \mid A_{2}$, then $T_{\tau} m=T_{\tau} \mu$.

Proof. Since $\mathscr{W}_{1}$ and $\mathscr{W}_{2}$ are bases for the closed sets for same topology, $\left(\mathscr{W}_{1}\right)_{\tau}=\left(\mathscr{W}_{2}\right)_{\tau}$ is the family of all closed sets for the topology. By Theorem 1.8, it is sufficient to prove that $m(W)=T_{\tau} \mu(W)$ for all $W \in \mathscr{W}_{1}$ : Hence fix. $W_{0} \in \mathscr{W}_{1}$ and $\dot{\varepsilon}>0$. Choose $f_{0} \in A_{1}$ with $\chi_{W_{0}} \leqslant f_{0}$ and $m\left(W_{0}\right)$ $>\varphi\left(f_{0}\right)-\varepsilon$. Then $\varepsilon+m\left(W_{0}\right)>\varphi\left(f_{0}\right)=\int_{X} f_{0} d T_{\tau} \mu \geqslant T_{\tau} \mu\left(W_{0}\right)$. Thus $T_{\tau} \mu\left(W_{0}\right)$ $\leqslant m\left(W_{0}\right)$ for all $W_{0} \in \mathscr{W}_{1}$.

Since $\mathscr{W}_{2}$ is base for the closed sets, $\mathscr{W}_{1} \subset\left(\mathscr{W}_{2}\right)_{\tau}$. Hence, since $T_{\tau} \mu$. is $\tau$-additive, there is $W_{1} \in \mathscr{W}_{2}$ with $W_{0} \subset W$, and $T_{\tau} \mu\left(W_{0}\right)+\varepsilon>\mu\left(W_{1}\right)$. Furthermore, there is $f_{1} \in A_{2}$ with $\chi_{W_{1}} \leqslant f_{1}$ and $\varphi\left(f_{1}\right)<\mu\left(W_{1}\right)+\varepsilon$. Hence, $T_{\tau} \mu\left(W_{0}\right)>\mu\left(W_{1}\right)-\varepsilon>\varphi\left(f_{1}\right)-2 \varepsilon=\int_{X} f_{1} d T_{\tau} m-2 \varepsilon \geqslant T_{\tau} m\left(W_{1}\right)-2 \varepsilon$ $\geqslant T_{\tau} m\left(W_{0}\right)-2 \varepsilon$. Since $\varepsilon>0$ was arbitrary, $T_{\tau} \mu\left(W_{0}\right) \geqslant T_{\tau} m\left(W_{0}\right)=m\left(W_{0}\right)$ for all $W_{0} \in \mathscr{W}_{1}$. The proof is complete.

We note that $\mathscr{W}$ is a normal base if $\mathscr{W}$ is paving of closed sets on $X$ which is a base for the closed sets of $X$ and satisfies: 
(i) If $G$ is a closed set in $X$ and if $x \in G^{c}$, then there are $W_{1}, W_{2} \in \mathscr{W}$ with $W_{1} \cap W_{2}=\varnothing$ and $x \epsilon W_{1}, G \subset W_{2}$.

(ii) If $W_{1}, W_{2} \in \mathscr{W}$ with $W_{1} \cap W_{2}=\varnothing$, then there are $\nabla_{1}, \nabla_{2} \in \mathscr{W}$ with $\nabla_{1} \cup \nabla_{2}=X$ and $W_{i} \subset \nabla_{i}^{o}$ for $i=1,2$.

We remark that a normal base $\mathscr{W}$ gives rise to a compactification $X_{\mathscr{W}}$ and if this compactification is $X_{A}$ (the compactification such that every element $f \in A$ can be extended to $C\left(X_{A}\right)$ and every element of $C\left(X_{A}\right)$ is such an extension), then $M(\mathscr{W})$ represents $A^{*}$ by $[4 ; 3.12]$.

THEOREM 2.2. Let $A_{1}, A_{2}$ be algebras on $X$ with $A_{2} \subset A_{1}$ and $\tau_{A_{1}}=\tau_{A_{2}}$; and let $\mathscr{W}_{1}$ and $\mathscr{W}_{2}$ be normal bases with $X_{A_{j}}$ the $\mathscr{W}_{j}$-compactification for $j=1,2$. Let $\left(\varphi_{i}\right)$ be a net in $\left(\mathcal{A}_{1}^{*}\right)^{+}$, let $\varphi \in\left(\mathcal{A}_{1}^{*}\right)^{+}$and assume that $\varphi_{i}(f) \rightarrow \varphi(f)$ for all $f \in A_{2}$. Then $\varphi_{i}(f) \rightarrow \varphi(f)$ for all $f \in A_{1}$ if either of the following two conditions hold.

(1) $\mathscr{W}_{2} \subset \mathscr{W}_{1}$.

(2) $\varphi_{i} \in\left(A_{1}^{*}\right)_{\tau}^{+}$for all $i$.

Proof. First assume that $\mathscr{W}_{2} \subset \mathscr{W}_{1}$. (That is, condition (1) holds.) Let $m_{i}, m \in M^{+}\left(\mathscr{W}_{1}\right)$ represent $\varphi_{i}$ and $\varphi$, respectively, and let $\mu_{i}, \mu \in M^{+}\left(\mathscr{W}_{2}\right)$ represent the restrictions of $\varphi_{i}$ and $\varphi$ to $A_{2}$. By Proposition $[4 ; 4.8]$ $m \in M_{\tau}^{+}\left(\mathscr{W}_{1}\right)$ and $\mu \epsilon M_{\tau}^{+}\left(\mathscr{W}_{2}\right)$. Fix $W_{0} \epsilon \mathscr{W}_{1}$ and $\varepsilon>0$. Since $\mathscr{W}_{2}$ is a normal base, there is a $W_{1} \in \mathscr{W}_{2}$ with $W_{0} \subset W_{1}$ and $\mu\left(W_{1}\right)<T_{\tau} \mu\left(W_{0}\right)+\varepsilon$. For all $i, m_{i}\left(W_{1}\right)=\inf \left\{\varphi(f): f \in A_{1}, \chi_{W_{1}} \leqslant f\right\} \leqslant \inf \left\{\varphi_{i}(f): f \in A_{2}, \quad \chi_{W_{1}} \leqslant f\right\}$ $=\mu_{i}\left(W_{1}\right)$. Using this together with Theorem [4;3.13] and Lemma 2.1 we obtain that,

$$
\limsup m_{i}\left(W_{0}\right) \leqslant \limsup m_{i}\left(W_{1}\right) \leqslant \limsup \mu_{i}\left(W_{1}\right)
$$

$$
\leqslant \mu\left(W_{1}\right)<T_{\tau} \mu\left(W_{0}\right)+\varepsilon=m\left(W_{0}\right)+\varepsilon .
$$

The -esult is now an immediate consequence of theorem $[4 ; 6.3]$.

Now assume that condition (2) holds. Again let $m_{i}, m \in M_{\tau}^{+}\left(\mathscr{W}_{1}\right)$ represent $\varphi_{i}$ and $\varphi$ and let $\mu_{i}, \mu \in M_{\tau}^{+}\left(\mathscr{W}_{2}\right)$ represent the restrictions of $\varphi_{i}$ and $\varphi$ to $A_{2}$. By Lemma $2.1, T_{\tau} \mu_{i}=T_{\tau} m$ and $T_{\tau} \mu=T_{\tau} m$. Fix $W_{0} \epsilon \mathscr{W}$ and $\varepsilon>0$. Choose $W_{1} \in \mathscr{W}_{2}$ such that $W_{0} \subset W_{1}$ and $0 \leqslant \mu\left(W_{1}\right)-m\left(W_{0}\right)$ $=T_{\tau} \mu\left(W_{1}-W_{0}\right) \leqslant \varepsilon$. Then by theorem $[4 ; 6.3]$ and Lemma 2.1

$\lim \sup m_{i}\left(W_{0}\right)=\lim \sup T_{\tau} \mu_{i}\left(W_{0}\right) \leqslant \lim \sup \mu_{i}\left(W_{1}\right) \leqslant \mu\left(W_{1}\right) \leqslant m\left(W_{0}\right)+\varepsilon$.

The result follows by another application of theorem $[4 ; 6.3]$.

CoRoLlaRY 2.3. Let $X$ be a compact Hausdorff space, $\left(m_{i}\right)$ a net of Borel measures on $X$ and $\mathscr{W}$ a base for the closed sets in $X$. Then $\left(m_{i}\right)$ converges weakly to a Borel measure $m$ if and only if $m_{i}(X) \rightarrow m(X)$ and limsup $m_{i}(W) \leqslant m(W)$ for all $W \in \mathscr{W}$.

We remark that in $\left[3\right.$; appendix(d)] we showed that $M_{\sigma}(\mathscr{W})$ is not weakly sequentially complete even when $\mathscr{W}$ is a normal base. (It is well known that if $A=C^{b}(\bar{X})$, then $M_{\sigma}(\mathscr{Z})$ is weakly sequentially complete.
See [8].) Thus we state the following

Problem. Let $\left(\varphi_{n}\right)$ be a sequence in $A_{\sigma}^{*}$ and assume that $\varphi_{n} \rightarrow \varphi$ in the $\sigma\left(A^{*}, A\right)$-sense. Does it necessarily follow that $\varphi \in A_{\sigma}^{*}$ ? If not, what conditions on the algebra will guarantee that this is so?

Lemana 2.4. Let $A$ be an algebra on $X$, and let $C^{b}$ denote the algebra

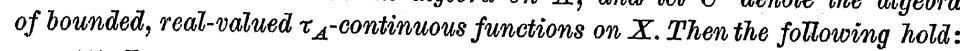

(1) Let $\mathscr{W}$ be a normal base for $\tau_{A}$ with $X_{A}$ the $\mathscr{W}$-compactification. Then there is a unique Riesz space isomorphism $T$ of $M_{\tau}(\mathscr{W})$ into $M_{\tau}\left(\mathscr{Z}\left(C^{b}\right)\right)$ which is a homeomorphism for the weak topologies.

(2) Let $\mathscr{W}=\mathscr{Z}(A)$, then the restriction map $S$ of $M_{\tau}\left(\mathscr{Z}\left(C^{b}\right)\right)$ to $\mathscr{F}(\mathscr{Z}(A))$ is a Riesz space isomorphism of $M_{\tau}\left(\mathscr{Z}\left(C^{b}\right)\right)$ onto $M_{\tau}(\mathscr{Z}(A))$ which is a homeomorphism for the weak topologies.

(1). The uniqueness is immediate from the fact that $L(X)$ is wealkly dense in $M_{\tau}(\mathscr{W})$ by Proposition $[4 ; 6.4]$. Since $\mathscr{W}$ is a normal base, $\mathscr{W}_{\tau}$ $=\left(\mathscr{Z}\left(O^{b}\right)\right)_{\tau}=\mathscr{F}$, the family of all $\tau_{\mathcal{A}^{-}}$-closed sets. Let $T_{1}$ be the map $T_{\tau}$ of $M_{\tau}(\mathscr{W})$ into $M_{\tau}(\mathscr{I})$ of Theorem 1.8 , and let $T_{2}$ be the corresponding map of $M_{\tau}\left(\mathscr{Z}\left(C^{b}\right)\right)$ onto $M_{\tau}(\mathscr{I})$. Let $T=T_{2}^{-1} \circ T_{1}$. Then $T$ is a Riesz space isomorphism which keeps $L(X)$ pointwise fixed. It is clear that $T^{-1}$ is continuous for the weak topologies. Let $\left(m_{i}\right)$ be a net in $M_{\tau}^{+}(\mathscr{W})$ and $m \in M_{\tau}^{+}(\mathscr{W})$. Assume that $m_{i} \rightarrow m$ weakly. We must show that $T m_{i} \rightarrow T m$.

Hence fix $Z_{0} \in \mathscr{Z}\left(C^{b}\right)$ and $\varepsilon>0$. Take $W_{0} \in \mathscr{W}$ with $Z_{0} \subset W_{0}$ and $m\left(W_{0}\right)<T_{1} m\left(Z_{0}\right)+\varepsilon$. Then by theorem $[4 ; 6.3]$

$$
\begin{aligned}
\limsup T m_{i}\left(Z_{0}\right) & =\limsup T_{1} m_{i}\left(Z_{0}\right) \leqslant \limsup T_{1} m_{i}\left(W_{0}\right) \\
& \leqslant \limsup m_{i}\left(W_{0}\right) \leqslant m\left(W_{0}\right)=T_{1} m\left(W_{0}\right) \\
& \leqslant T_{1} m\left(Z_{0}\right)+\varepsilon=T m\left(Z_{0}\right)+\varepsilon .
\end{aligned}
$$

It now follows by theorem $[4 ; 6.1]$ that $T m_{i} \rightarrow T m$ weakly. The proof of $(2)$ is similar to that of (1) except that we use $[4 ; 6.1]$ in place of $[4 ; 6.3]$. See also $[1, p .12]$ and [8].

Using Lemma 2.4, we can now obtain several facts about weak compactness as consequences of results which are known in $C^{b}(X)^{*}$. (See [5].)

THEOREM. 2.5. Let $A$ be an algebra on $X$ and assume that $\left(X, \tau_{A}\right)$ is metrizable as a separable metric space. Then a set $B \subset\left(A_{\tau}^{*}\right)^{+}$is relatively wealcly compact if and only if it is relatively weakly sequentially compact.

Probf. Let $B^{\prime} \subset M_{\tau}^{+}(\mathscr{Z}(A))$ be the set of measures which represent the elements of $B$ according to theorem $[4 ; 4.8]$. By (1) in Lemma 2.4, $B^{r}$ is relatively weakly compact if and only if $S\left[B^{\prime}\right]$ is relatively weakly compact in $M_{\tau}^{+}\left(\mathscr{Z}\left(C^{b}\right)\right)$. But by theorem 27 of $[5, \mathrm{p} .76], S\left[B^{\prime}\right]$ is relatively weakly compact if and only if $S\left[B^{\prime}\right]$ is relatively weakly sequentially compact. Again by Lemma 2.4, this is equivalent to $B^{\prime}$ being weakly sequentially compact. The proof is complete. 
THEOREM 2.6. Let $A$ be an algebra on $X$, and let $B \subset\left(A_{\tau}^{*}\right)^{+}$. If $B$ is relatively weakly countably compact in $\left(A_{\tau}^{*}\right)^{+}$, then $B$ is relatively wealily compact in $\left(A_{\tau}^{*}\right)^{+}$.

Proof. It is enough to prove the theorem in the special case, $A=C^{b}(X)$. The general result then follows from Lemma 2.4 as in the proof of Theorem 2.5 above. But if $A=C^{b}$, then $M_{\sigma}(\mathscr{Z})$ is complete for the Mackey topology $m\left(M_{\sigma}(\mathscr{Z}), O^{b}\right)$. (Indeed, it is shown in [3] that $M_{0}$ is complete for a topology $e^{b}$ for which the dual of $M_{\sigma}$ is $C^{b}$. Hence it is complete for the Mackey topology.) It then follows from Eberlein's theorem that if $B \subset M_{\sigma}(\mathscr{Z})$ is relatively weakly countably compact, then it is relatively weakly compact. The proof is complete.

Remark. We have shown above that any relatively wealkly sequentially compaict subset of $M_{\sigma}\left(\mathscr{Z}\left(C^{b}\right)\right)$ is necessarily relatively weakly compact.

We note from [4] that for a paving $\mathscr{W}$, a set $S \subset X$ is $\mathscr{W}$-compact if for every filter $\mathscr{U} \subset \mathscr{W}$ with $S \cap U \neq \varnothing$ for all $U \in \mathscr{U}$, then $\cap\{S \cap U$ : $\left.U_{\epsilon} \mathscr{U}\right\} \neq \varnothing$.

Definimion 2.7. Let $\mathscr{W}$ be a paving on $\not$. A set $B \subset M(\mathscr{W})$ is tight if $\sup \{\|m\|: m \epsilon B\}<\infty$ and if for every $\varepsilon>0$, there is a $\mathscr{W}$-compact set $W_{0} \in \mathscr{W}_{\tau}$ such that $|m|(W) \leqslant \varepsilon$ for all $W \in \mathscr{W}$ with $W \cap W_{0}=\varnothing$ and all $m \in B$.

It is clear that the mapping $S$ of Lemma 2.4 preserves tight sets. Hence using Lemma 2.4 and Theorem 31 of $[8$, p. 66], we obtain the following weakened version of Prochorov's theorem.

THEOREM 2.8. Let $A$ be an algebra and assume that $\left(X, \tau_{A}\right)$ is locally compact or that $\left(X, \tau_{\mathcal{A}}\right)$ is metrizable with a complete metric. Then $B \subset M_{\tau}^{+}(\mathscr{Z}(A))$ is relatively $\sigma\left(A^{*}, A\right)$-compact if and only if it is tight.

We conclude this paper with a last application to obtain a generalization of a known result (see for example $[5 ; 5.1(d)]$ ). We denote the set of tight elements of $\boldsymbol{M}(\mathscr{W})$ by $\boldsymbol{M}_{t}(\mathscr{W})$.

THEOREM 2.9. Let $\mathscr{W}$ be a normal base with compactification $X_{A}$ or let $\mathscr{W}=\mathscr{Z}(A)$ for $A$ an algebra on $X$. If $m \in M_{t}(\mathscr{W})$, then there is a unique compact regular Borel measure $\mu$ on $X$ such that $\mu \mid \mathscr{F}(\mathscr{W})=m$ and $\mu^{+}, \mu^{-}$ $\epsilon M_{\tau}\left(\mathscr{W}_{\tau}\right)$.

Proof. We note that the hypothesis implies the $\tau_{A}$-compact setis are $\mathscr{W}$-compact and conversely. By $[4 ; 5.6] m \in M_{t}(\mathscr{W})$ is in $M_{\tau}(\mathscr{W})$. Therefore there exist unique $\mu^{+}, \mu^{-} \epsilon M_{\tau}\left(\mathscr{W}_{\tau}\right)$ such that $\mu^{+} \mid \mathscr{F}(\mathscr{W})=m^{+}$ and $\mu^{-} \mathscr{F}(\mathscr{W})=m^{-}$by 1.7. Since $\mathscr{W}$ is a base for the $\tau_{A}$ closed setsof $X, \mathscr{W}_{v}$ is the paving of all closed sets so that $\mu^{+}, \mu^{-}$are Borel measures on $X$.

Finally, since $m$ is tight, for any $\varepsilon>0$ there is a $\mathscr{W}$-compact set $W_{0}$ such that $|m|(W)<\varepsilon$ if $W \cap W_{0}=\varnothing$. Since $W_{0}$ is $\tau_{A}$-compact and $\mathscr{W}$ is a basis for the $\tau_{A}$ closed sets, it follows that for any closed set $F$ with $F \cap W_{0}$ $=\emptyset$, there is a $W \in \mathscr{W}$ such that $F \subset W$ and $W \cap W_{0}=\emptyset$. Consequently, $|\mu|(F) \leqslant|\mu|(W)=|m|(W)<\varepsilon$ so that $|\mu|$ is compact regular.

\section{References}

[1] P. Billingsley, Oonvergence of Probability Measures, Wiley, New York 1968.

[2] L. Drewnowski, Decompositions of set functions, Studia Math. 68 (1973), pp. 23-48.

[3] R. B. Kirk, Algebras of bounded real-valued functions I, II, Nederl. Akad. Wetensch Proc. Sev. A 75, Indg. Math. 34 (1972), pp. 443-463.

[4] - and J. A. Crenshaw, A generalized topological measure theory. Trans. Amer. Math. Soc. 207 (1975), pp. 189-217.

[5] F. D. Sentilles, Bounded continuous functions, Trans. Amer. Math. Soc. 168 (1972), pp. 311-336.

[6] M. Sion, Outer measures with values in a topological group, Proc. London Math. Soc. 19 (1969), pp. 89-106.

[7] Tim Traynor, A General Hewit-Yosida Decomposition, Canad. J. Math. 26 (1972), pp. 1164-1169.

[8] V. S. Varadarajan, Measures in topological spaces (Russian), Mat. Sbornik N. S. 55 (1961), pp. 33-100. Translated in Amer. Math. Soc. Transl. (2) 72 (1965), pp. 161-228.

Received October 23, 1974

Revised version May 30,1975

(907) 